\title{
Promotional Efforts of Pharmaceutical Industries on Prescribing Pattern of Antibiotics among Medical Doctors in Nepal
}

\author{
Prabhat Ranjan Napit ${ }^{l}$
}

\begin{abstract}
Promotional efforts of pharmaceutical industries on prescribing pattern of antibiotics among doctors in Nepal" has been chosen as a topic for research to resolve certain queries among physicians' behavior, particularly drug prescribing patterns as the influence of promotional approaches of pharmaceutical industry. The main objective of this research is to ascertain the various promotional activities of pharmaceutical industries of Nepal and find out its effects on prescribing pattern among physician in case of antibiotics along with rationality of prescription.

For this study, data are collected both from primary as well as secondary sources. 225 sample size is taken from 4 districts (Kathmandu, Lalitpur, Bhaktapur and Kavre) to represent the country and convenient quota sampling is used to select the sample. The data are collected manually as the questionnaire is designed. From the data analysis, it is found that there is significant relationship between promotional efforts towards qualification of doctors and cost of antibiotics is considered as prioritized reason as well. And there is no significant relationship between patient compliance/ better quality among alternatives/ obligation (as a compensation of efforts)/ availability/ appearances as prioritized reason and promotional activities on qualification of doctors. Furthermore, there is significant relationship between influence of detailing/gift/ monetary incentives/ trip and tours on prescribing pattern and rational prescription of antibiotics and no significant relationship between influence of samples and bonus on prescribing pattern and rational prescription of antibiotics.
\end{abstract}

Keywords: pharmaceutical industries; prescribing pattern; rational prescription; primary survey and descriptive statistics

\footnotetext{
Mr. Napit is a Ph.D. Scholar in Basic Pharmaceutical Sciences in University of Louisiana at Monroe, USA. He would like to express his gratefulness to Dr. Ram Kumar Phuyal for his insightful suggestions, guidance, and tips on shaping this paper in the present form. He would also like to appreciate to Mr. Sohan Babu Khatri and Mr. Prabhat Uprety for their cooperation and supports. Similarly, he is thankful to two anonymous reviewers for their valuable comments and suggestions.
}

Corresponding Email: prabhat.napit@gmail.com 
Napit: Promotional Efforts of Pharmaceutical Industries ...

\section{Introduction}

The pharmaceutical industry applies a large proportion of its resources to marketing and advertising operations (Petryna and Kleinman 2006). One central issue in the debate about the pharmaceutical industry has been whether it is primarily driven by innovation or marketing. Information about promotional expenditures of the pharmaceutical companies is rarely available and is questioned by the public health expert and Consumer Rights Activists. A majority of critics of the pharmaceutical policy in developing countries seem to favor a drastic regulation of health services, including pharmaceuticals (Van der Geest 1984). Their basic idea is that the profit maximization is by definition pathogenic because it puts profit before people. The structure of relations between manufacturers and the supply chain (the importer, wholesaler and retailer), between manufacturer and prescriber, involves something more like reciprocal access to guarded resources (Lakoff 2006). Prescription drug promotion practices that involve giving financial grants and valuable items to doctors and retailers are common in Nepal (Subedi 2001; GAPN 2007; Thapa 2007). The Government of Nepal, Department of Drug Administration (DDA), has developed and released Guidelines on Ethical Promotion of Medicine-2007 with the objective to enhance ethical promotion of medicine to support and encourage the improvement of health care through the rational use of medicine and discourage unethical practices. The guidelines, however, could not properly be implemented due to the conflict of interests among various stakeholders. This research describes interests of specific stakeholders i.e. doctors, focusing particularly on the issue of bonuses, gifts or any other incentives. This is not only interesting for producers, distributors and retailers, but also one of the major concerns of the nation and the public.

There has been increased interest in the relationship between the pharmaceutical industry and physicians and whether contact impacts the fiduciary relationship between physicians and their patients. More than $10 \%$ of revenue is spent each year by pharmaceutical companies in promotion and marketing. Drug companies allocate an estimated certain amount for physician per year to be spent by pharmaceutical marketing representatives. How is this money being spent, and for what purpose? The influence of pharmaceutical company marketing on physician behavior, particularly drug prescribing patterns, has not been extensively studied, and what data exists is limited to self-report of behavior as opposed to observation of physician behavior in relation to interactions with specific drug companies. "Promotional efforts of Pharmaceutical Industries on prescribing pattern of antibiotics among doctors in Nepal" has been chosen as a topic for research to solve queries; 
- Which of the promotional activities of antibiotics have significant role in prescribing patterns among doctors?

- Why do the promotional activities influence the prescribing pattern among doctors?

- Is prescription of antibiotics rational though there is a high influence of promotional efforts?

It is the purpose of this study to investigate the extent of exposure of pharmaceutical representatives, the attitudes of each of the groups toward this interaction, the appropriateness of receiving gifts, and to establish whether prescribing of marketed drugs is related to exposure to the drug marketers in context of Nepal. The aim of the research is to study the significance of promotional efforts in prescription pattern among doctors, effective promotional activity and rational prescription of medicine. These are compared in respect to several promotional activities exposure and prescription of antibiotics is observed. The medicine category chosen to have definable amounts of marketing associated with each drug over a month-long period and will therefore be ranked accordingly. By understanding their frequency of prescribing certain drugs, we can evaluate the association with exposure to drug marketing. Hence, to determine the effects of promotional efforts on prescription pattern; identification of the promotional activities in prescribing patterns among doctors and examination of the most effective activity among several to influence the prescribing pattern are focused.

Pharmaceutical marketing efforts are also problematic if they influence health professionals to prescribe drugs that are not the best choices for their patients. Of course, prescribers often do not know what the best choice will ultimately be for each patient and must try different therapies. Problems arise when health professionals prescribe drugs with incomplete knowledge of identified risks, a situation that can arise when pharmaceutical marketing efforts fail to adequately disclose side effects or potential drug interactions. (Busy healthcare providers' lack of time to keep up with the latest medical literature also contributes to this problem, and academic detailing efforts can help address this.) Marketing efforts can also contribute to excessive enthusiasm for new drugs that leads some prescribers to stop prescribing older products, even though older drugs may have a better balance of risks and benefits for some patients.

Hence, this research drives the physical practitioners to implement the rational use of antibiotics which will definitely play the crucial towards the progress of health status of country. This also helps to direct pharmaceutical companies to conduct promotional activities in viable limit to make aware of brand of their generic drugs rather than to interfere the rational prescription pattern. 
204 Napit: Promotional Efforts of Pharmaceutical Industries ...

\section{Review of Literature}

Lexchin(1993) has tried to analyze the effect of three types of interaction between physicians and the pharmaceutical industry (company-funded clinical trials, company-sponsored continuing medical education, CME) and information for physicians supplied by pharmaceutical detailers on orientation and quality of clinical trials, content of CME courses and physicians' prescribing behavior. Similarly, Acemoglu,Beyhun,Calikogluand Vancelik(2007) have conducted the research to study the commercial sources of information which are considered to be of greater influence than scientific sources on general practitioners' (GPs) prescribing behavior in under developed and developing countries. The study has aimed to determine the self-reported impact of pharmaceutical promotion on the decision-making process of prescription of GPs in Eastern Turkey. In addition, Alssageer and Kowalski (2012) have conducted the research on the purpose of the investigation to examine the frequency of pharmaceutical company representative (PCR) interactions with doctors in Libya.

Jureidini and Mansfield (2001) have conducted the research to increase awareness amongst psychiatrists and trainees of the effects of pharmaceutical promotion and to stimulate careful evaluation of the relationships between psychiatry and the pharmaceutical industry. Moreover, Adeyemi, Oreagba and Oshikoya (2011) have tried to examine the approach in the direction of unethical/inappropriateness and lead towards irrational prescribing. Dearth of information on impact of pharmaceutical drug promotion on prescribing behavior of doctors in developing African countries has necessitated this study. Therefore, it is aimed to determine the sources of drug information for doctors working in a teaching hospital in Nigeria and to assess the self-reported impact of the sources on their prescribing behavior. Likewise, Farooq, Goel and Goyal (2016) have jointly put an effort to analyze their sales and hence, their shareholder value. These corporations need to make their product with a better formulation than those already available and stand out from their adversaries. This is achieved with the help of rigorous promotion of the drug to the prescribers. Medical Representatives (MRs) or Pharmaceutical Sales Representatives (PSRs) advertise to the doctors with the help of eye catching visual presentation, citing various benefits and advantages.

Barbalee, Cheryl, Michael and Samuel (2006) have made an effort to analyze whether family physicians (FPs) who distribute free sample medications are more likely to prescribe those medications than physicians who do not. By the same token, Hurley,Lane and Stafford (2014) have conducted the research describing the relationship between the availability of free prescription drug samples and dermatologists' prescribing patterns on a national scale. This can help inform policy guidelines on the use of free samples in a physician's office. Its objective is to 
investigate the relationships between free drug samples and dermatologists' local and national prescribing patterns and between the availability of free drug samples and prescription costs. Likewise, Cidda, Krishna, Nalini, Parmar and Pavani (2012) have concerned oneself to examine the prescribing behavior of physicians, evaluate average cost per prescription, educate the patients regarding their medication and health status and to promote rational use of drugs for achieving better patient outcomes.

Hall,Tett and Nissen (2006) have made an effort to analyze the knowledge, attitudes, and behaviors of Australian family physicians regarding the use of sample prescription medications. Apart from this, Akhtar, Akram, Hameed, Hussain, Malik, Parveen, Riaz, Saeed, Siddiqi and Yasin (2011) have exerted oneself to investigate the importance and influence of promotional tools used by pharmaceutical industry on prescribing behaviors of doctors/consultants. They have also exerted to establish comparison between doctors/consultants versus medical representatives and consultant versus doctors with an auxiliary of difference between local and multinational company's representative. Similarly, Alhilali and Mikhael (2014) have made an effort to analyze the interaction between physicians and medical representatives (MRs) through gift offering, a common cause for conflicts of interest for physicians that negatively influence prescribing behaviors of physicians throughout the world. This study has aimed to evaluate the interaction between MRs and Iraqi specialist physicians through the acceptance of MRs gifts and the effect of such acceptance on physician's prescribing patterns.

Cajetan, Emecheta, Emmanuel, Geraldine, Ikenna, Linus, Obinna and Uchenna (2010) have made an attempt to examine drug promotions used in multifaceted approaches incorporating hospital and office detailing by marketing representatives. Very few studies exist on their influence on doctors' prescription pattern in Nigeria. They have examined the scope and effects of marketing strategies on the prescription habits of doctors in Enugu, South East Nigeria. And Zaki (2014) has made an attempt to examine an adopting a randomized, multiple site and cross-sectional survey study, questionnaires $(\mathrm{n}=250)$ completed by physicians and pharmacists to investigate the exposure, acceptance or skepticism of Saudi physicians/pharmacists to drug promotion as well as their perception of the appropriateness of gifts and to check if they have any teaching/training about dealing with medical representatives (MRs) and Pharma promotion.

Ahmed, Mohiuddin, Nahar, Rashid and Shuvro (2015) have investigated to study which is conducted as part of a larger study to explore the status of governance in health sector in 2009. Moreover, Codi, Khan and Manimekalai (2014) have exerted oneself to evaluate and compare the prescribing pattern of private practitioners and physicians of a tertiary care teaching hospital in a semi urban area and detect their 
rationality. And Mikhael (2014) has conducted the research to evaluate the interaction between medical representatives (MRs) and physicians and the effect of such interaction on physician prescribing behavior, according to the site of view for Iraqi pharmacists.

Zahrani (2014) has made an attempt to analyze the assess of drug representatives' influence on physician's prescription, to assess physician's attitudes toward drug representative and to study other factors that may affect the prescribing behavior of physicians. Furthermore, Hugget, Maio, Monaghan, Rich and Warrier (2010) have exerted oneself to judge systematic review and assess towards the influence of the pharmaceutical industry on physician prescribing. Also Khosru and Sultana (2011) have made an effort to analyze the promotional strategies followed by the pharmaceutical companies, attitudes and responses of physicians towards these promotional activities and influence of using gifts as promotional materials on the prescribing behavior of the physicians.

\section{The Methodology}

The study has been planned to find out significance of promotional efforts towards prescription of antibiotics among doctors in Nepal. So, the research is Applied, Quantitative and Analytical type. The research is applied because theory, equation, statistical tools used in the study is existing tools. The research is also quantitative type because data are quantified, and analysis of population is done on behalf of sample.

\subsection{The conceptual model}

The research is based on the paper "Effect of promotional strategies of pharmaceutical companies on doctors' prescription pattern in South East Nigeria". This study has demonstrated the most commonly employed promotional strategies by pharmaceutical companies in South East Nigerian medical doctors. As much as $60 \%$ of doctors admit to being influenced by promotional strategies. Nevertheless, despite all forms of marketing and drug promotion, the socioeconomic status of the patient remains a key consideration in the prescription of medication. There is a still a need for more large scale prospective studies to further ascertain the impact factor of such marketing practices.

With reference to various models developed, promotional efforts of pharmaceutical industries on prescribing pattern of antibiotics among doctors is one among them for the study purpose. For it, relation between independent variables with dependent variables is conducted. The model developed is presented below: 
Independent Variables

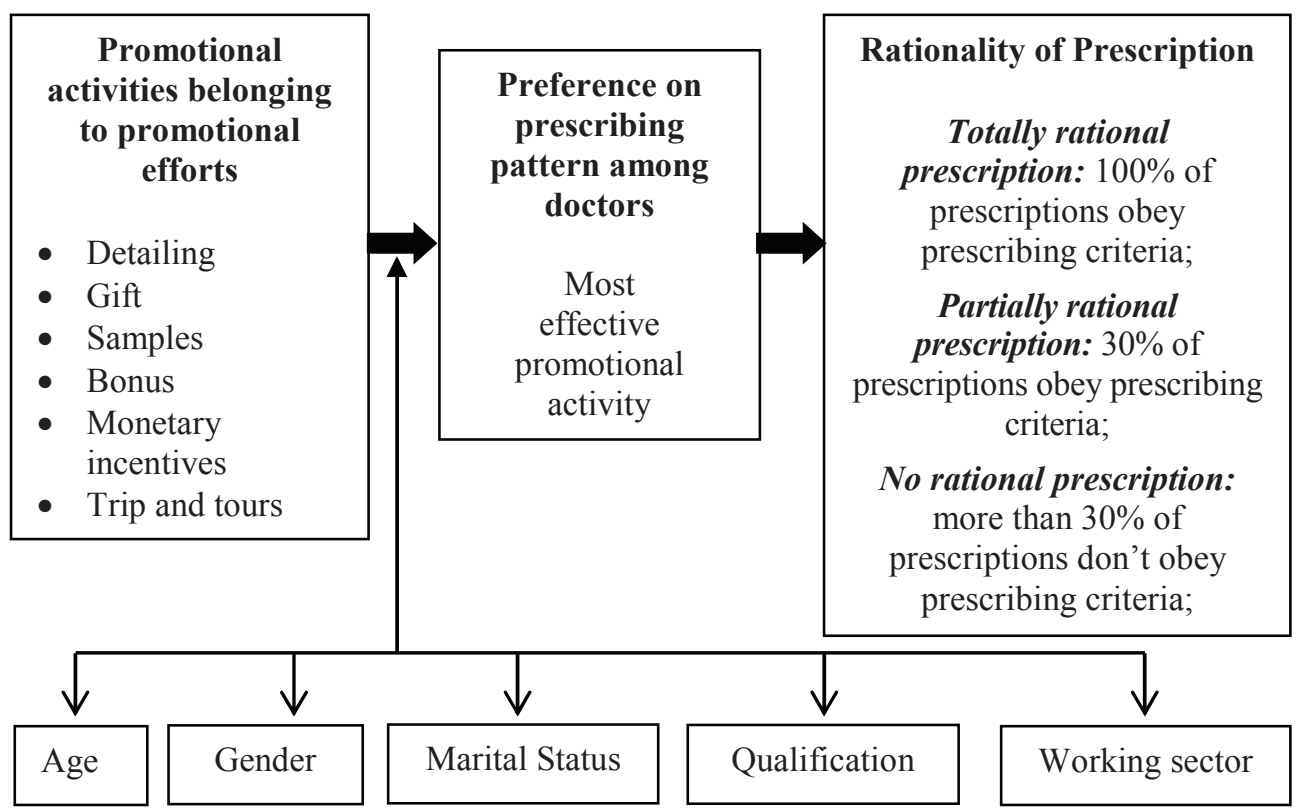

\section{Demographic Variables}

Figure 1. Conceptual Framework

The specified model for research is;

Functionally $\mathrm{Q}=\mathrm{f}(\mathrm{x} i \mathbf{i})$ where, $\mathrm{Q}=$ Preference on prescribing pattern among Doctors $\mathrm{x}=$ Detailing, Gift, Samples, Bonus, Monetary incentives, Trip and tours $i=$ Kathmandu, Bhaktapur, Lalitpur and Kavre

\section{Dependent Variables:}

Preference on prescribing pattern among Doctors; Rationality of prescription

\section{Independent Variables:}

Promotional activities regarding promotional efforts:

Detailing

Gift

Samples

Bonus

Monetary incentives

Trip and tours

\section{Demographic Variables:}

Age, Gender, Marital Status, Qualification, Hospital/Clinic is included as demographic variables. They are measured as: 
208 Napit: Promotional Efforts of Pharmaceutical Industries ...

Age: Below 30, 30 - less than 40, 40- less than 50, 50 and above

Gender: Male and Female

Qualification: MBBS, MD/MS, Above MD/MS

Marital Status: Single, Married

Working sector: Hospital, Clinic, Primary Health Centre (PHC), Health Post (HP)

\title{
3.2 The data
}

The research data is both primary as well as secondary. The questionnaire is filled by several quotas of sample and these questionnaires are used for analysis which is primary source of data and secondary reliable data and information are also included. For the research, population is all the Marketing Representatives (MRs) of the country since they are the best option to get all the information for the research. For sample, non-random sampling technique with quota sampling which is convenient is chosen. The sample size for the completion of research is around 225. Regarding the sample size determination, average 20 percent coverage of doctors by pharmaceutical industry in each district is considered. The sample size is somehow sufficient for representing the population. So, the samples are categorized into four different quotes according to geographical distribution; Kathmandu (87), Bhaktapur (44), Lalitpur (62), Kavre (32).

\section{Sample size of 4 districts}

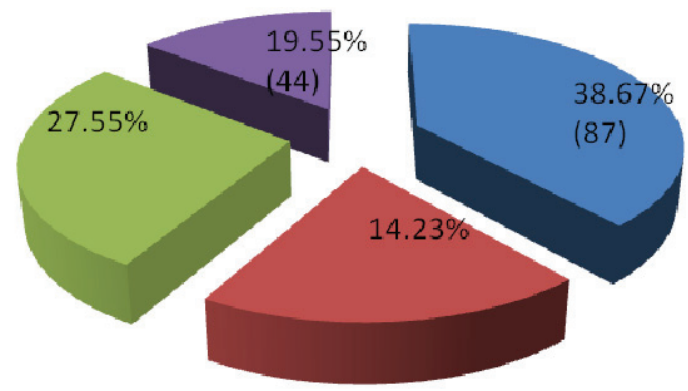

\author{
Kathmandu \\ Bhaktapur \\ - Lalitpur
}

Kavre

\subsection{Data analysis methods}

As the questionnaire is collected, editing and coding process are conducted. Later Excel and SPSS software are used for data management. Both descriptive and inferential analysis is conducted using Excel and SPSS software. In descriptive analysis;Mean, Median, Mode, Correlation are included while in inferential analysis; T- tests, One way ANOVA (F- test), Chi- square test are presented. 


\section{The Results and Discussion}

This chapter presents the results obtained from the data analysis and research methodology. The data set is used to examine the assumptions of factor analysis. The results of various frequencies, ANOVA and cross tabulation are presented, and the null hypotheses are tested. The results are discussed in terms of their relation to each of the relevant research objectives. The data and information collected from the respondents are presented, interpreted, and analyzed according to response of respondents on the field survey.

\subsection{Description of sample}

According to the respondents, 45 (20\%) doctors have MBBS degree, $123(54.66 \%)$ have qualification of above MD/MS and 57 (25.34\%) have qualification above MD/MS. The number of doctors working as full time in Hospital is $175(77.77 \%)$ and Clinic is $50(22.23 \%)$. The cross checking of prescription replicates that 50 $(22.23 \%)$ doctors prescribe the medicine rationally, $169(75.11 \%)$ doctors' prescription shows partially rational and $6(2.66 \%)$ doctors' show no rational prescription. Ranking of variables is in ascending order. They are as follows: Influence of Gift on prescribing Pattern with mean 2.63, Influence of Samples with mean 2.75, Influence of Detailing with mean 2.78, Influence of Bonus with mean 3.89, Influence of Monetary incentives with mean 4.12 and Influence of trip and tours with mean 4.78 .

\section{Qualification of doctor classification}

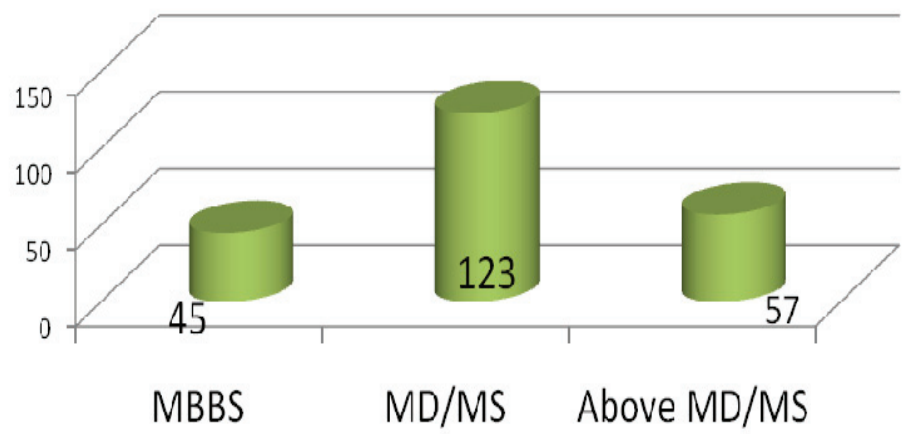




\section{Rational prescription of antibiotics classification}

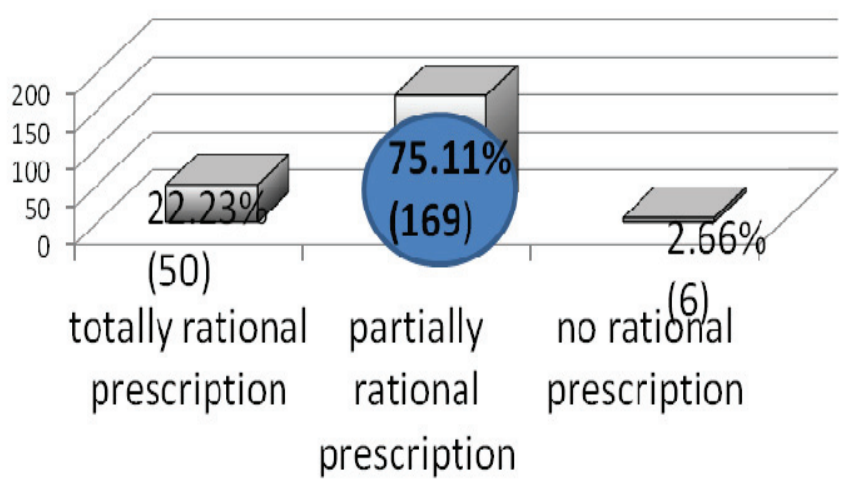

\subsection{Analysis of results}

From the analysis, we can say that 29 doctors (64.45\%) with MBBS qualification prefer Patient Compliance as prioritized reason, 64 doctors $(52.04 \%)$ with MD/MS qualification prefer it and 37 doctors (64.91\%) prefer it. 37 doctors $(82.23 \%)$ with MBBS qualification prefer Cost of antibiotics as prioritized reason, 59 doctors (47.96\%) with MD/MS qualification prefer it and 30 doctors (64.91\%) prefer it. 17 doctors $(37.77 \%)$ with MBBS qualification prefer Better quality among alternatives as prioritized reason, 68 doctors (55.28\%) with MD/MS qualification prefer it and 30 doctors $(52.63 \%)$ with qualification above MD/MS prefer it. 2 doctors $(4.44 \%)$ with qualification of MBBS prefer Obligation (as a compensation of efforts) as prioritized reason, 10 doctors $(8.14 \%)$ prefer it and 4 doctors $(7.02 \%)$ with qualification above MD/MS prefer it. 8 doctors (17.77\%) with qualification of MBBS prefer Availability as prioritized reason and 37 doctors, 32 doctors (26.02\%) with qualification MD/MS prefer it and 8 doctors $(14.04 \%)$ with qualification above MD/MS prefer it. 1 doctor $(2.22 \%)$ with the qualification of MBBS prefers Appearances as prioritized reason and 44 doctors, 5 doctors $(4.06 \%)$ with the qualification of MD/MS prefer it and 2 doctors (3.5\%) with the qualification of above MD/MS prefer it.

Similarly, $87,32,33,27,7,39$ are responding from first to sixth respectively as influence of detailing on prescribing pattern and 13, 4, 6, 13,1, 13 are number of total rational prescription. $38,87,42,40,13,5$ are responding as influence of gifting and $9,18,8,6,7,2$ are number of total rational prescription. 58, 60, 36, 32, 29, 10 are responding as influence of samples and $15,12,5,7,6,5$ are number of total rational 
prescription. 8, 8, 69, 73, 48, 19 are responding as influence of bonus and 3, 1, 23, 11, 8,4 are number of total rational prescription. $22,21,30,32,73,47$ are responding as influence of Monetary incentives and 7, 10, 0, 10, 16, 7 are number of total rational prescription. 13,17, 15,21,54,105 are responding as influence of trip and tours and 4, $5,8,3,11,19$ are number of total rational prescription.

Additionally, 217 have responded 'yes' to the question 'Are promotional activities the effective means in the pharmaceutical marketing?' and 8 have responded 'no'. Most of them agree on these factors; Promotional activities remind the brand name of antibiotics among several alternatives, Promotional activities surely enhance on prescribing pattern among doctors, Promotional activities are a practicable means of marketing in Nepal, Promotional efforts have significant role to increase sales. We can conclude this since all factors have mean value less than 3(Neutral) and those who have answered 'yes' to the question have mean value even less than 2 .

\section{Concluding Remarks}

This research provides relationship of dependent variables (detailing, gift, samples, bonus, monetary incentives, trip and tours) on prescribing pattern among physician in case of antibiotics along with the rationality of prescription. Likewise, the relationship between demographic variables; age, gender, qualification, marital status and working sector of doctors with the prescribing pattern of antibiotics is also determined. Similarly, the prioritized reasons while prescribing among physicians i.e. Patient Compliance, Cost of Medicine, and Better Quality among alternatives, Obligation (as a compensation of efforts), availability, and appearances are also analyzed.

The test shows that there is no significant relationship between 'Qualification of the doctors' and 'Patient Compliance as prioritized reason'/ 'Better quality among alternatives as prioritized reason'/ 'Obligation (as a compensation of efforts) as prioritized reason'/ 'Availability as prioritized reason'/ 'Appearances as prioritized reason'. On the other hand, there is significant relationship between 'Qualification of the doctors'/ 'Cost of antibiotics as prioritized reason'. The study also concludes that there is no significant relationship between 'Promotional activities as effective means in the pharmaceutical market' and 'Promotional activities remind the brand name of antibiotics among several alternatives'/ 'Promotional activities surely enhance on prescribing pattern among doctors'/ 'Promotional activities are a practicable means of marketing in Nepal'/ 'Promotional efforts have significant role to increase sales'. Similarly, the test exhibits that there is significant 
relationship between 'Influence of Detailing/ Gift/ Monetary Incentives/ Trip and Tours on prescribing pattern' and 'Rational prescription of antibiotics'. On the next side, there is no significant relationship between 'Influence of Samples/ Bonus on prescribing pattern' and 'Rational prescription of antibiotics'.

The findings of this study have significant implications for prescribing pattern of antibiotics among physicians. The promotional efforts of pharmaceutical industries have ultimately direct impact on sales of their products. These findings provide an insight into the characteristics and practices of marketing. Since promotional efforts have crucial role on prescribing pattern, these efforts should enhance to uplift the sales of domestic pharmaceuticals products within as well as outside the country. And doctors should concern on rational prescription of medicine since it is inevitable things in human life. Rational prescription of medicine will play great role on uplifting health sector and reducing drug resistance.

\section{References}

Ahmed, S.M., Mohiuddin, M., Nahar, N., Rashid, S.F. \& Shuvro, M.I. (2015).Qualitative insights into promotion of pharmaceutical products in Bangladesh: How ethical are the practices? BioMed Central Journal, Vol. 16(80), 1-9.

Akhtar, P., Akram, T.S., Hameed, A.,Hussain, S., Malik, F., Parveen, G., Riaz, H., Saeed, T., Siddiqi, A. \& Yasin, F. (2011). Relevant influence of promotional tools by pharmaceutical industry on prescribing behaviors of doctors: A crosssectional survey in Pakistan. African Journal of Pharmacy and Pharmacology, Vol. 5(13), 1623-1632.

Alhilali, D.N. \& Mikhael, E.M. (2014).Gift acceptance and its effect on prescribing behavior among Iraqi specialist physicians. Pharmacology \& Pharmacy, Vol. 5, 705-715.

Alssageer, M.A. \& Kowalski, S.R. (2012). A survey of pharmaceutical company representative interactions with doctors in Libya. Libyan Journal of Medicine, Vol. 7(18556), 1-20.

Barbalee, S., Cheryl, P., Michael, A. \& Samuel N.F. (2006).Effects of using free sample medications on the prescribing practices of family physicians. Journal Am Board Fam Med, Vol. 19(5), 443-449.

Cajetan, O., Emecheta O., Emmanuel, A., Emmanuel, E., Geraldine, U., Ikenna, O., Linus, O., Obinna, O. \& Uchenna, I. (2010).Effect of promotional strategies 
of pharmaceutical companies on doctors' prescription pattern in South East Nigeria.TAF Preventive Medicine Bulletin, Vol. 9(1), 1-6.

Cidda, M., Krishna, T.R., Nalini, M., Parmar, M.Y. \& Pavani, V. (2012).Study of prescribing patterns of common health problems. International Journal of Pharmacy and Biological Sciences, Vol. 2(4), 22-31.

Codi, S., Khan, S. \& Manimekalai, K. (2014). Prescribing practices of nonteaching general practitioners of private clinics and physicians of a tertiary care teaching hospital: A comparative cross sectional study. International Journal of Medical Research \& Health Sciences, Vol. 4(1), 72-77.

Farooq, M., Goel, R. \& Goyal, M. (2016).Effect of promotional literature on the prescribing habits of practitioners. International Journal of Medical and Dental Sciences, Vol. 5(1), 1104-1111.

Hall, K.B., Tett, S.E. \& Nissen, L.M. (2006). Perceptions of the influence of prescription medicine samples on prescribing by family physicians.US National Library of Medicine National Institutes of Health, Vol. 44(4), 383387.

Hugget, K., Maio, A., Monaghan, M.S., Rich, E. \& Warrier, R. (2010). Effect of drug sample availability on physician prescribing behavior: A systematic review. Clinical Reviews and Opinions, Vol. 2(4), 41-48.

Hurley, M.P., Lane, A.T. \& Stafford, R.S. (2014). Characterizing the relationship between free drug samples and prescription patterns for acne vulgaris and rosacea. US National Library of Medicine National Institutes of Health, Vol. $150(5), 487-493$.

Jureidini, J. \& Mansfield, P. (2001). Does drug promotion adversely influence doctors' abilities to make the best decisions for patients? Australasian Psychiatry Journal, Vol. 9(2), 95.

Khosru, K.H. \& Sultana, S. (2011). Practice of using gifts as promotional materials for marketing of pharmaceutical products in Bangladesh: A survey conducted on general physicians and representatives from pharmaceutical companies. Stamford Journal of Pharmaceutical Sciences, Vol. 4(2), 13-18.

Lexchin, J. (1993). Interactions between physicians and the pharmaceutical industry: what does the literature say? Canadian Medical Association Journal, Vol. 149 (10), 1401-1407. 
214 Napit: Promotional Efforts of Pharmaceutical Industries ...

Mikhael, E.M. (2014). Evaluating the effect of medical representative on physician prescribing pattern in Iraq. Asian Journal of Pharmaceutical and Clinical Research, Vol. 7(1), 222-223.

Oshikoya, K.A., Oreagba, I. \& Adeyemi, O. (2011).Sources of drug information and their influence on the prescribing behavior of doctors in a teaching hospital in Ibadan. Nigeria Pan African Medical Journal, Vol. 9(13), 1-9.

Vancelik, S., Beyhun, N.E., Acemoglu, H. \& Calikoglu, O. (2007). Impact of pharmaceutical promotion on prescribing decisions of general practitioners in Eastern Turkey. Bio Medical Centre Public Health, Vol. 7(122),1-8.

Zahrani, H.S.A. (2014). The impact of pharmaceutical promotions on primary health care physicianl's prescribing behavior in KAMC in central region. International Journal of Medical Science and Public Health, Vol. 3(3), 358-361.

Zaki, N.M. (2014). Pharmacists' and physicians' perception and exposure to drug promotion: A Saudi study. US National Library of Medicine National Institutes of Health, Vol. 22(6), 528-536. 